\title{
Inhibition of return and visual search: How many separate loci are inhibited?
}

\author{
JANICE J. SNYDER \\ University of Alberta, Edmonton, Alberta, Canada \\ and \\ ALAN KINGSTONE \\ University of British Columbia, Vancouver, British Columbia, Canada
}

\begin{abstract}
Using a novel sequential visual search paradigm Danziger, Kingstone, and Snyder (1998) demonstrated that inhibition of return (IOR) can reside at three spatial locations. In the present study, we extended the work of Danziger et al. by investigating whether there is a limit to the number of locations that can be inhibited in a sequential visual search task. Our study revealed that IOR can be measured at a minimum of five locations. The magnitude of the IOR effect was largest at the most recently searched location and declined from there in an approximately linear fashion. Two models that can account for our data are presented.
\end{abstract}

The acquisition of stimulus information in the environment is facilitated for items that are attended. Behaviorally, this is reflected in a reduced response time (RT) and/ or error rate for attended items, relative to unattended items. A classic demonstration is provided by the Posner cuing paradigm (Posner, 1980). Two empty squares flank a central fixation box. The task for the subject is to maintain central fixation and to respond with a keypress as quickly and as accurately as possible when a target stimulus, such as an asterisk, appears in one of the two squares. Just prior to target onset, one of the two squares is brightened briefly. Importantly, this brightening of a square does not predict where the target will appear. The typical result is that when the delay between the brightening of the box and onset of the target is $300 \mathrm{msec}$ or less, RT is shorter if a target appears in the cued location (the box that brightened), rather than in the uncued location (the box that did not brighten). This RT difference between the cued and the uncued locations is attributed to attention being drawn reflexively to the cued location, which results in a processing advantage for a target that appears there (Jonides, 1981; Müller \& Rabbitt, 1989; Posner, 1980).

Interestingly, the opposite pattern of results is obtained if the delay between the cue and the target exceeds 300 $500 \mathrm{msec}$ (Maylor, 1985; Posner \& Cohen, 1984). Now,

This research was supported by graduate student awards to J.J.S. from the Natural Sciences and Engineering Research Council of Canada (NSERC) and the Alberta Heritage Foundation for Medical Research (AHFMR), and by research grants to A.K. (NSERC Grant 170077 and AHFMR Grant 00134). The authors thank Ray Klein, Jay Pratt, an anonymous reviewer, and Jeremy Wolfe for helpful comments on earlier versions of this manuscript. Correspondence concerning this article should be addressed to J. J. Snyder or A. Kingstone, Department of Psychology, University of Alberta, Edmonton, AB T6G 2E9, Canada. (e-mail: jsnyder@ualberta.ca or alan.kingstone@ubc.ca).
RT is longer when a target appears at the cued location, as compared with the uncued location. Posner and Cohen suggested that if a target does not appear soon after attention is committed to the cued location, attention is withdrawn from that location. When an ensuing target is presented at this previously attended/cued location, RT is delayed because attention is "inhibited from returning" to the cued location. Posner and Cohen speculated that this bias against returning attention to previously attended locations could serve an important ecological role, because it would improve the efficiency of searching complex environments.

Klein (1988) was the first to test this idea. He had subjects perform an attentionally demanding search for a target stimulus. Occasionally, when search was completed, Klein presented a probe item at a location that either was or was not likely to have been searched. The subjects responded to the probe as quickly as possible. Consistent with the notion that inhibition of return (IOR) persists at locations that have been inspected, Klein discovered that RT to the probe stimulus was longer when it appeared at a location that was likely to have been attended during search. Unfortunately, subsequent attempts to replicate this result have failed, seriously undermining the notion that IOR serves to improve search efficiency (see Klein \& Taylor, 1994; Wolfe \& Pokorny, 1990; but see Klein \& MacInnes, 1999).

Pratt and Abrams (1995) struck another serious blow to the notion that IOR serves to facilitate visual search. They found that when multiple locations were cued sequentially, IOR dwelled only at the most recently cued location. The implication of this finding is that when multiple locations in the environment must be scanned, IOR plays little or no role in improving search efficiency.

However, Tipper, Weaver, and Watson (1996) recently suggested that the paradigm used by Pratt and Abrams 
(1995) may have been inappropriate for testing whether IOR dwells at multiple locations. Tipper et al. pointed out that Pratt and Abrams (1995) presented subjects with only two possible target locations. As a result, the subjects may have inhibited only the most recently cued location, because inhibiting both locations would have had the detrimental effect of increasing RT overall. In support of this proposal, Tipper et al. showed that when there were four possible target locations and three were cued, IOR was found to dwell at each location.

Abrams and Pratt (1996) noted, however, that the subjects in the Tipper et al. (1996) study might have grouped each of the cued locations into one contiguous region of inhibited space (see also Wright \& Richard, 1996). Consistent with this idea, Abrams and Pratt (1996) showed that when three locations were cued in a manner that could preclude spatially contiguous grouping, IOR resided at only the most recently cued location.

In summary, the empirical evidence did not provide compelling support for the idea that IOR can dwell at multiple locations in visual space. And thus, there was little reason to think that IOR serves to improve search efficiency (see also a study by Horowitz \& Wolfe, 1998, that raises further problems for the functional role of IOR in search). However, more recently, a study by Danziger, Kingstone, and Snyder (1998) has shown that IOR can reside at three spatially noncontiguous locations in a sequential visual search task. In the Danziger et al. investigation, there were five possible target locations marked by squares in the periphery. The locations were organized around an imaginary circle with a fixation stimulus at the center. Prior to target onset, one, two, or three of these target locations could be cued in sequence, or target onset might occur without any precue. An important difference between this investigation and all previous IOR studies was that the number of cues preceding target onset varied randomly from trial to trial. Therefore, on any given trial, when a stimulus event occurred in the periphery, the subjects presumably had to direct their attention to the stimulus to determine whether it was a cue or a target. Danziger et al. hypothesized that when the peripheral event was found to be a cue and not a target, IOR would be laid down at the inspected location.

The results of Danziger et al. (1998) demonstrated that IOR dwelled at each of the cued locations. That is, IOR was observed at one cued location when there was one cue, at two cued locations when there were two cues, and at three cued locations when there were three cues. In addition, it was discovered that this multiple IOR occurred whether or not the cued locations were spatially contiguous, demonstrating that spatial grouping was not responsible for the effect. Finally, and most important for the present investigation, it was found that the magnitude of the IOR effect declined from the most recently cued location.

Given that IOR declines across cued locations, it seems likely that there is a finite number of locations that can be inhibited in sequential visual search. Determining that value has important implications, because it could establish the boundary condition beyond which IOR can no longer be expected to improve search efficiency. For instance, if IOR can dwell at no more than three locations, then when there are more than three locations to be searched, it is reasonable to expect that attention will begin to return to a previously inspected location.

The aim of the present investigation was to determine the number of locations at which IOR can be measured in a sequential visual search task. As a first pass at this issue, we modified the Danziger et al. (1998) paradigm by increasing the number of possible target locations from five to eight and by doubling the number of possible cues from three to six.

\section{METHOD}

\section{Subjects}

Thirty experimentally naive University of Alberta undergraduate students participated in this experiment for course credit. All the subjects reported normal or corrected-to-normal vision.

\section{Apparatus and Stimuli}

The stimuli were displayed on a computer monitor. The stimulus display consisted of a black background with eight dark-gray outline boxes measuring $1.5^{\circ}$ of visual angle (va) on each side and placed equidistantly around an imaginary circle. The circle's radius, from its center to the center of each box, measured $6.5^{\circ} \mathrm{va}$. The first box was located at an angle of $18^{\circ}$ va to the right of the vertical meridian, and the last box was located at an angle of $18^{\circ}$ va to the left of the vertical meridian. A light-gray central fixation cross $\left(0.5^{\circ} \mathrm{va}\right)$ marked the center of the imaginary circle. The cues were light-gray outline boxes that appeared over the dark-gray outline boxes. The target was a light-gray asterisk $\left(0.5^{\circ} \mathrm{va}\right)$. Response latencies were recorded by the computer and measured in milliseconds. Figure 1 illustrates a six-cue trial in which the target appeared at the third-last-cued location.

\section{Procedure}

The subjects were seated in a dimly lit room, $57 \mathrm{~cm}$ from the display screen. They were informed that the cue(s) did not predict where the target would occur. The subjects were instructed to maintain fixation on the central cross and to respond as quickly and as accurately as possible by pressing the space bar when a target appeared. Eye monitoring was not incorporated, because we had demonstrated repeatedly in our laboratory that subjects will not make eye movements to simple target stimuli in this task even when provided with the opportunity to do so (see also Posner, 1980). Each trial began with a $100-\mathrm{msec}$ warning tone. Following a 500-msec stimulus onset asynchrony (SOA), either a target appeared or a peripheral box was brightened for $100 \mathrm{msec}$ (the attentional cue). If a cue occurred, there was a 500-msec SOA, and then a target appeared or a different box was brightened. Up to six peripheral cues could precede target onset, with the SOA between all the stimuli (cues and target) fixed at $500 \mathrm{msec}$. The location of each cue was randomly selected, with the restriction that the same location was not cued twice in the same trial. On catch trials, seven cues appeared, and no target was presented.

\section{Design}

The subjects participated in two 50 -min sessions separated by 2 or 3 days. Each session consisted of 600 trials divided into 12 blocks of 50 trials, with a rest break separating each block. Thus, each subject received a total of 1,200 test trials. Of these 1,200 trials, 24 were zero-cue trials (the target appeared following the warning tone), and 24 were catch trials (the target never occurred). The remaining 1,152 trials were divided equally among the six different peripheral cue conditions (i.e., one-cue, two-cue, three-cue, four- 


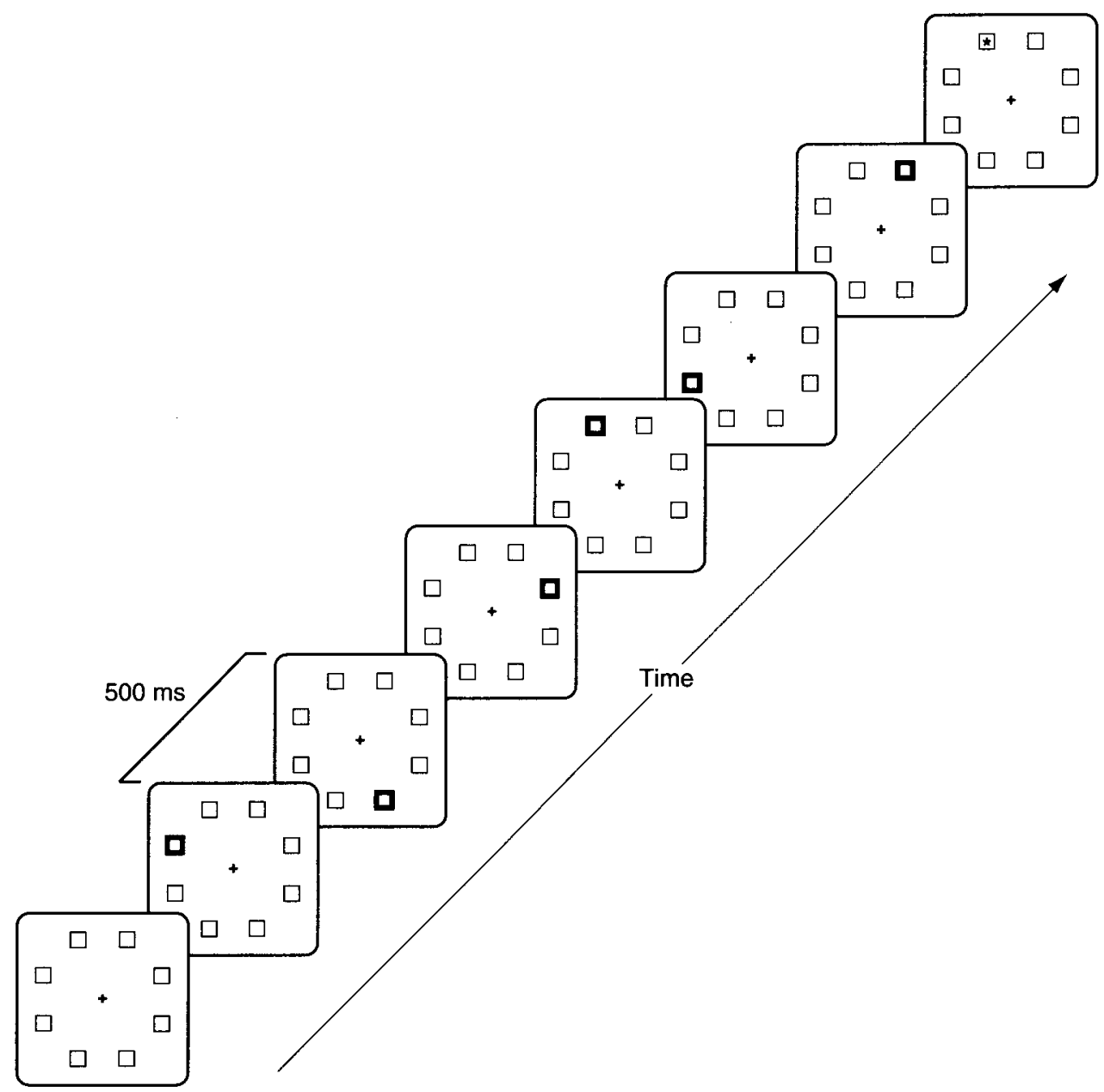

Figure 1. The sequence of events on a trial with six peripheral cues preceding target onset (the asterisk). All cue durations were $100 \mathrm{msec}$, and all stimulus onset asynchronies were $\mathbf{5 0 0} \mathbf{m s e c}$. In this trial, the target appears at the third-last location that was cued.

cue, five-cue, and six-cue conditions), resulting in 192 trials per cue condition. Each block of trials contained approximately one zerocue trial and one catch trial. The cues did not predict target location, and the target could occur equiprobably at any of the eight possible locations. On trials in which one location was cued prior to target onset (i.e., on one-cue trials), the target would appear 24 times at the cued location and 24 times at each of the seven uncued locations. Similarly, for the six-cue trials, the target would appear 24 times at each of the six cued locations and 24 times at each of the two uncued locations. Cue conditions were randomly selected.

For each of the two test sessions, the subjects first received 10 practice trials. All the trials were terminated when a keypress response was executed or $1,500 \mathrm{msec}$ after the last cue event, whichever came first. Response feedback was provided, with a 200 -msec error tone sounding when the subjects responded prior to target onset or when they failed to respond to the target. The error tone was easily distinguished from the warning tone.

\section{RESULTS}

Our goal was to determine the number of locations that can exhibit IOR. Before presenting our data, it is critical that our terminology is clear. When only one cue occurred prior to target onset and the target occurred at the cued location, we refer to this cued location as oneback from target onset. When two cues occurred in sequence prior to target onset and the target occurred at the location that was cued just prior to target onset, we again refer to this location as the one-back location. If the target occurred at the second-last location that was cued, we refer to this cued location as the two-back location. A similar progression occurs for three-cue, four-cue, fivecue, and six-cue trials. The target location illustrated in Figure 1 is a cued three-back location, following a sequence of six cues.

A separaie repeated measures analysis of variance (ANOVA) was performed for each of the cued-back locations (one-back through to six-back). For one-back through five-back location trials, a two-factor ANOVA was corducted with location (cued $N$-back vs. uncued) and number of cues as factors. For the six-back location trials, a one-factor ANOVA was conducted with target lo- 
Table 1A

\begin{tabular}{|c|c|c|c|c|c|}
\hline Cued Back Location & Source & $d f$ & $M S_{\mathrm{e}}$ & $F$ Ratio & $p$ Value \\
\hline \multirow[t]{3}{*}{ One-back } & Target location & 1 & 317.59 & 64.84 & $<.001$ \\
\hline & Number of cues & 5 & 295.31 & 99.09 & $<.001$ \\
\hline & Target location $\times$ number of cues & 5 & 185.29 & 1.44 & $>.2$ \\
\hline \multirow[t]{3}{*}{ Two-back } & Target location & 1 & 171.05 & 54.99 & $<.001$ \\
\hline & Number of cues & 4 & 229.58 & 48.16 & $<.001$ \\
\hline & Target location $\times$ number of cues & 4 & 151.35 & $<1$ & $>.4$ \\
\hline \multirow[t]{3}{*}{ Three-back } & Target location & 1 & 145.31 & 23.48 & $<.001$ \\
\hline & Number of cues & 3 & 174.49 & 22.99 & $<.001$ \\
\hline & Target location $\times$ number of cues & 3 & 136.23 & 2.86 & $<.05$ \\
\hline \multirow[t]{3}{*}{ Four-back } & Target location & 1 & 128.84 & 14.77 & $<.001$ \\
\hline & Number of cues & 2 & 145.27 & 10.11 & $<.001$ \\
\hline & Target location $\times$ number of cues & 2 & 105.72 & $<1$ & $>.8$ \\
\hline \multirow[t]{3}{*}{ Five-back } & Target location & 1 & 160.27 & 9.64 & $<.01$ \\
\hline & Number of cues & 1 & 162.51 & $<1$ & $>.7$ \\
\hline & Target location $\times$ number of cues & 1 & 173.33 & $<1$ & $>.4$ \\
\hline Six-back & Target location & 1 & 141.15 & $<1$ & $>.5$ \\
\hline
\end{tabular}

Table 1B

Mean Reaction Time (RT, in Milliseconds) and Mean Inhibition of Return (IOR) Effects in Milliseconds for Target Location and Number of Cues

\begin{tabular}{lccccccc}
\hline & \multicolumn{6}{c}{ Number of Cues } & \\
\cline { 2 - 7 } Target Location & 1 & 2 & 3 & 4 & 5 & 6 & IOR Effects \\
\hline Uncued & 432 & 408 & 394 & 383 & 373 & 374 & \\
One-back & 441 & 429 & 410 & 396 & 390 & 388 & 15 \\
Two-back & & 417 & 402 & 393 & 389 & 385 & 11 \\
Three-back & & & 399 & 384 & 386 & 384 & 8 \\
Four-back & & & & 388 & 379 & 381 & 7 \\
Five-back & & & & 382 & 379 & 7 \\
Six-back & & & & & 376 & 2 \\
\hline
\end{tabular}

Note-For each target location, IOR effects were based on the mean cued RT minus the mean uncued RT across the same number of cues.

cation (six-back vs. uncued) as a factor. Note that if IOR is present at a cued location, RT at a cued location should be longer than that at an uncued location. ANOVA results are presented in Table $1 \mathrm{~A}$, and RT performance is presented in Table 1B.

As is shown in Table 1A, a main effect of target location was observed for cued one-back, two-back, threeback, four-back, and five-back locations, indicating that IOR occurred at each of the cued locations. That is, RT at the cued locations was longer than RT at the uncued locations. The IOR effect at the cued six-back location, however, was not reliable. There was a main effect for number of cues for cued one-back, two-back, three-back, and four-back locations, with overall RT becoming shorter as the number of cues increased from one to four. A similar warning signal effect was observed by Danziger et al. (1998) and reflects the fact that the temporal uncertainty regarding when a target will occur is reduced as the number of cues is increased. The main effect of number of cues was not significant for the cued five-back and six-back locations, indicating that the subjects were maximally prepared to respond to the target. There was no interaction between target location and number of cues for cued one-back, two-back, and four-back locations, indicating that the IOR effect did not vary with the number of cues in these conditions. An interaction between target location and number of cues did occur for the cued three-back analysis. Mean contrasts revealed the source of the interaction: a significant IOR effect at the threeback location when five or six cues preceded target onset $[F(3,87)=18.78$ and 11.02 , respectively; all $p s<.01]$, but only a marginally significant IOR effect on three-cue trials $\left[F(3,87)=3.60, M S_{\mathrm{e}}=136.23, p<.07\right]$, and a nonsignificant IOR effect on four-cue trials $(F<1)$.

In summary, IOR effects were observed at the cued one-back through five-back locations (ranging from 15 to $7 \mathrm{msec}$; see Table 1B), but not at the six-back location $(2 \mathrm{msec})$. It is our position, however, that the 2-msec IOR effect at the six-back location is also real. When a linear regression analysis of the IOR effects reported above (one-back through six-back) was conducted, we discovered that cued back location accounted for $89.5 \%$ of the IOR variance $\left[F(1,4)=34.26, M S_{\mathrm{e}}=2.60, p<.01 ;\right.$ see Figure 2]. ${ }^{\prime}$

The slope of this linear regression line was $-2.3 \mathrm{msec}$, indicating that the IOR effect decreased by about $2.3 \mathrm{msec}$ 


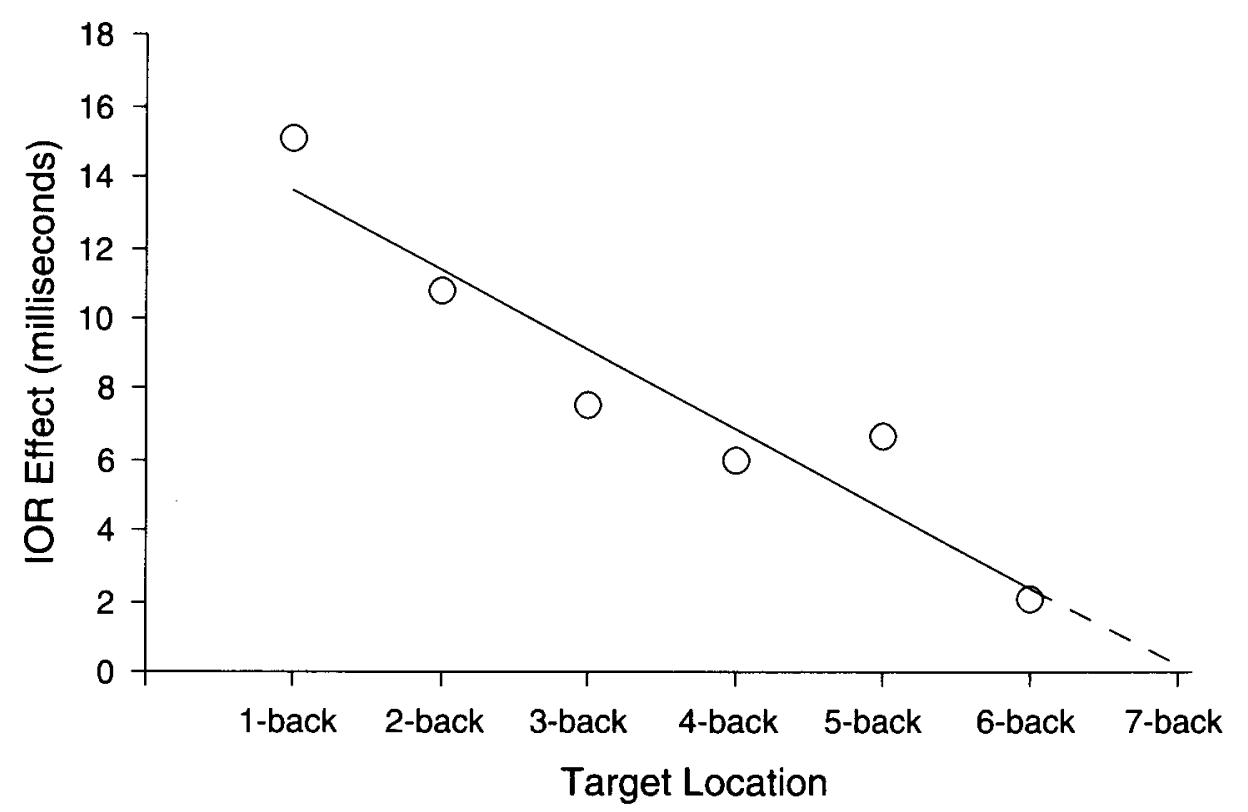

\begin{abstract}
Figure 2. Inhibition of return (IOR) effects (cued RT minus uncued RT) in milliseconds for cued one-back through six-back locations used in the regression analysis. The $y$-intercept is $15.9 \mathrm{msec}$, and the slope across cued back locations is $-2.3 \mathrm{msec}$. The regression line has been extrapolated to reveal the hypothetical location (cued seven-back) at which the IOR effect would reach 0 msec.
\end{abstract}

per location. Given that the decline in IOR across locations is approximately linear, one can predict the size of the IOR effect at the six-back location from the slope $(-2.3 \mathrm{msec})$ and intercept $(15.9 \mathrm{msec})$ of the regression line $[15.9 \mathrm{msec}-(2.3 \mathrm{msec} \times 6)=2.1 \mathrm{msec}]$. This predicted IOR effect of $2.1 \mathrm{msec}$ at the six-back location matches remarkably well with the 2 -msec effect that was observed, so it is reasonable to consider that the 2-msec effect is real. Probably, this $2-\mathrm{msec}$ effect was not statistically reliable because of a lack of power in the ANOVA. Recall that the nature of our present experimental design dictated that there would be an equal number of one-cue, two-cue, three-cue, four-cue, five-cue, and six-cue sequences preceding target onset. This means that on only one of these six trial sequences could a target occur at a six-back location (i.e., the six-cue condition). In terms of trial number, this means that there were 24 trials per subject for a six-back location and 144 trials per subject for a one-back location.

\section{Response Accuracy}

Overall error rates were consistently low. False alarms (responses prior to target onset) occurred on $0.7 \%$ of all the trials. Misses (failure to respond to a target within $1,500 \mathrm{msec}$ ) occurred on $0.2 \%$ of the trials. Anticipations (RT less than $100 \mathrm{msec}$ following target onset) occurred on $0.008 \%$ of the trials. ANOVAs revealed that the error rates never varied significantly as a function of whether a target appeared at a cued versus an uncued location (all $\left.F_{\mathrm{s}}<1.01\right)$.

\section{DISCUSSION}

The present research determined that, in a sequential visual search paradigm, (1) IOR magnitude declines in an approximately linear fashion from the most recently cued location and (2) IOR effects can be measured reliably at five locations and possibly at six locations.

At first glance, Pylyshyn's (1989) FINST theory would appear to provide a good account of these findings. First, FINST theory assumes that spatial tagging can occur for only four to five locations in the visual field, and this is consistent with the number of locations at which IOR was measured reliably. Second, FINST theory assumes that spatial tagging occurs in environmental coordinates (Pylyshyn, 1989), and IOR is considered to occur in environmental rather than retinotopic coordinates (Maylor, 1985; Pratt \& Abrams, 1997). And finally, FINST theory assumes that spatial tagging can be goal directed (Trick \& Pylyshyn, 1993), and IOR is known to be sensitive to goaldirected processes (e.g., IOR does not occur at a location that is likely to receive a target; Posner \& Cohen, 1984; Tipper et al., 1996).

But is FINST theory consistent with our finding that the magnitude of IOR declines across cued back locations in an approximately linear manner? Because FINST theory postulates that spatial tagging operates in an allor-none manner, one might argue that a linear decline in IOR across cued back locations should not occur until all of the tags have been exhausted - that is, the magnitude of IOR should not decline until the fourth or fifth lo- 
cation is cued. However, FINST theory can easily accommodate a linear decline in IOR if it is simply assumed that locations tagged early in a cue sequence are increasingly more likely to have their tags stolen (interference) or lost (temporal decay and/or interference) as additional cues are presented (see Schmidt, Fisher, \& Pylyshyn, 1998 , for data supporting the view that FINST may behave in this manner). ${ }^{2}$ Thus FINST theory can accommodate our finding that IOR can be measured reliably at only five locations and that the IOR effect declines in an approximately linear fashion.

Determining whether the monotonic decline in IOR across cued locations is due to additional cues or merely to the passage of time is a goal for future research. The role played by additional cues in the decline of IOR can be tested by holding the time interval between the first cue and the appearance of the target constant while varying the number of cues. Conversely, the role played by temporal decay can be tested by holding the number of cues preceding target constant while varying the time interval between the first cue and the appearance of the target. We are now beginning to conduct research based on these two lines of investigation.

It is important to note, however, that although the present study does not discriminate whether the decline in IOR across locations is due to the effects of cuing additional locations or the passage of time, Danziger et al. (1998) can provide some insight. The results of Danziger et al. suggest that the decline in IOR is due to the introduction of additional cues, rather than to the passage of time. If IOR declines as a function of time alone, then according to the present study, the IOR effect reaches $0 \mathrm{msec}$ by the seven-back location (see the extrapolated line in Figure 2), where the cue-target interval is $3,500 \mathrm{msec}$. In the Danziger et al. (Experiment 3 ) investigation, the subjects were presented with up to three sequential peripheral cues, each separated by an SOA of $1,600 \mathrm{msec}$. Thus, the cue-target interval was $1,600 \mathrm{msec}$ for a one-back location, 3,200 msec for a two-back location, and 4,800 msec for a three-back location. A temporal decay account of IOR predicts that Danziger et al. would find IOR at the two-back location, but not at the three-back location. Importantly, however, Danziger et al. observed that IOR was present at each of the three locations $(11 \mathrm{msec}$ at the three-back location). Therefore, these data suggest that the addition of cued locations, rather than the passage of time, is crucial for the decline in IOR across successively cued locations.

If the addition of cues does prove to be the key factor underlying the decline in IOR across cued locations, it is worth noting that the FINST mechanism is not the only way to account for the data. Such a finding would also be consistent with a mechanism in which a number of items in the environment could be ordered and held in a queue of limited size for attentional servicing. According to this account, when a cued location is searched and no target is found, the searched location is entered into the priority queue (see Yantis \& Johnson, 1990, and Yantis \& Jones,
1991, for a similar mechanism with respect to attentional priority in visual search). Position within the queue represents the inhibitory value of each particular item. Items that have most recently entered the queue have the highest inhibitory value (i.e., the highest priority), and items further into the queue have progressively less inhibition (i.e., less priority). The present study suggests that up to five or six items might be held in the queue at any given time.

In summary, there are at least two possible mechanisms that can account for our finding that IOR can be measured at multiple locations, with the IOR effect declining across cued locations: a FINST-like inhibitory tagging mechanism and an inhibitory priority queue mechanism. One goal for future research will be to discriminate between these two accounts and to assess the importance of number of cues versus temporal decay in the decline of IOR. Another important goal for future investigation will be to determine whether the IOR measured in our sequential visual search paradigm serves a functional role when subjects are presented with a traditional conjunction visual search (e.g., Treisman \& Gelade, 1980; Wolfe, 1998). To date, all we can say with confidence is that, in the sequential visual search paradigm, IOR can be measured at a minimum of five locations, with the magnitude of IOR declining from the most recently cued location in an approximately linear fashion.

\section{REFERENCES}

Abrams, R. A., \& Pratt, J. (1996). Spatially-diffuse inhibition affects multiple locations: A reply to Tipper, Weaver, and Watson (1996). Journal of Experimental Psychology: Human Perception \& Performance, 22, 1294-1298.

DANZiger, S., KingSTONE, A., \& SNyder, J. J. (1998). Inhibition of return to successively stimulated locations in a sequential visual search paradigm. Journal of Experimental Psychology: Human Perception \& Performance, 24, 1467-1475.

Horowitz, T., \& WolfE, J. M. (1998). Visual search has no memory. Nature, 394, 575-577.

JoNIDES, J. (1981). Voluntary versus automatic control over the mind's eye's movement. In J. [B.] Long \& A. [D.] Baddeley (Eds.), Attention and performance $I X$ (pp. 187-203), Hillsdale, $\mathrm{NJ}$ : Erlbaum.

KLEIN, R. [M.] (1988). Inhibitory tagging system facilitates visual search. Nature, 334, 430-431.

KLEIN, R. M., \& MACINNES, W. J. (1999). Inhibition of return is a foraging facilitator in visual search. Psychological Science, 10, 346-352.

KLEIN, R. M., \& TAYLOR, T. L. (1994). Categories of cognitive inhibition with reference to attention. In D. Dagenbach \& T. H. Carr (Eds.), Inhibitory processes in attention, memory, and language (pp. 113150). San Diego: Academic Press.

MAYLOR, E. A. (1985). Facilitatory and inhibitory components of orienting in visual space. In M. I. Posner \& O. S. M. Marin (Eds.), Attention and performance XI (pp. 189-204). Hillsdale, NJ: Erlbaum.

Müller, H. J., \& RabbitT, P. M. A. (1989). Reflexive and voluntary orienting of visual attention: Time course of activation and resistance to interruption. Journal of Experimental Psychology: Human Perception \& Performance, 15, 315-330.

POSNER, M. I. (1980). Orienting of attention. Quarterly Journal of Experimental Psychology, 32, 3-25.

POSNER, M. I., \& Cohen, Y. (1984). Components of visual orienting. In H. Bouma \& D. G. Bouwhuis (Eds.), Attention and performance X: Control of language processes (pp. 531-556). Hillsdale, NJ: Erlbaum.

PratT, J., \& Abrams, R. A. (1995). Inhibition of return to successively cued spatial locations. Journal of Experimental Psychology: Human Perception \& Performance, 21, 1343-1353. 
Pratt, J. \& Abrams, R. A. (1997, November). Retinal coding of inhibited eye movements to recently attended locations. Poster presented at the 38th Annual Meeting of the Psychonomic Society, Philadelphia.

PYLYSHYN, Z. (1989). The role of location indexes in spatial perception: A sketch of the FINST spatial-index model. Cognition, 32, 65-97.

Schmidt, W. C., Fisher, B. D., \& Pylyshyn, Z. W. (1998). Multiplelocation access in vision: Evidence from illusory line motion. Journal of Experimental Psychology: Human Perception \& Performance, 24, 505-525.

TipPeR, S. P., Weaver, B., \& Watson, F. L. (1996). Inhibition of return to successively cued locations: Commentary on Pratt and Abrams (1995). Journal of Experimental Psychology: Human Perception \& Performance, 22, 1289-1293.

Treisman, A. M., \& Gelade, G. (1980). A feature-integration theory of attention. Cognitive Psychology, 12, 97-136.

TRICK, L. M., \& PYLYSHYN, Z. W. (1993). What enumeration studies can show us about spatial attention: Evidence for limited capacity preattentive processing. Journal of Experimental Psychology: Human Perception \& Performance, 19, 331-351.

WOLFE, J. M. (1998). What can a million trials tell us about visual search? Psychological Science, 9, 33-39.

WOLFE, J. M., \& PokoRnY, C. W. (1990). Inhibitory tagging in visual search: A failure to replicate. Perception \& Psychophysics, 48, 357-362.
Wright, R. D., \& Richard, C. M. (1996). Inhibition-of-return at multiple locations in visual space. Canadian Journal of Experimental Psychology, 50, 324-327.

YANTIS, S., \& JohnSON, D. N. (1990). Mechanisms of attentional priority. Journal of Experimental Psychology: Human Perception \& Performance, 16, 812-825.

YANTIS, S., \& JONES, E. (1991). Mechanisms of attentional selection: Temporally modulated priority tags. Perception \& Psychophysics, 50 , 166-178.

\section{NOTES}

1. We greatly appreciate the comments from Ray Klein and an anonymous reviewer for suggesting the importance of observing overall trends in the data, rather than relying heavily on the nonsignificant $F$ value at the six-back location.

2. We sincerely thank Ray Klein for his insightful comments on this issue.

(Manuscript received March 23, 1998; revision accepted for publication April 21, 1999.) 